\title{
7 Liste der Interviewpartner (anonymisiert)
}

Nr. Bezeichnung

1 Mitarbeiterin

2 Nanotechnologieverantwortliche

3 Nanotechnologieverantwortlicher

4 Nanotechnologieverantwortlicher

$5 \quad$ Leitungsmitglied

6 Nanotechnologieverantwortlicher

7 Nanotechnologieverantwortlicher

8 Nanotechnologieverantwortliche

9 Leitungsmitglied

10 Nanotechnologieverantwortlicher

11 Chemierechtspezialist

12 Nanotechnologiespezialistin

13 Leitungsmitglied

14 Pharmazeutikaspezialist

15 Leitungsmitglied

16 Ökonom

17 Chemikalienspezialist

18 Vertreter

19 Mitinitiator der NNI

20 Chemikalienrechtsspezialist

21 Nanotechnologierechtsspezialistin

22 Dialogspezialistin

23 Sozialwissenschaftler

24 Leitungsmitglied

25 Nanotechnologieverantwortlicher

26 Nanotechnologieverantwortlicher
Organisation

Datum

Environmental Defense, Boston

2.1.2008

Woodrow Wilson International Center for Scholars

Woodrow Wilson International Center for Scholars

Occupational Safety and Health Administration (OSHA)

Rice University

21.1.2008

DuPont, Amerian Chemistry Council (ACC)

15.1.2008

15.1.2008

15.1.2008

22.1.2008

22.1.2008

22.1.2008

White House Office of Science and Technology Policy

Meridian Institute

23.1.2008

Food and Drug Administration (FDA)

Boston University

24.1.2008

28.1.2008

Bergerson \& Campbell P.C.

28.1.2008

MIT Museeum

30.1.2008

Harvard Business School

19.2.2008

Woodrow Wilson International Center for Scholars

4.3.2008

Harvard University

5.3 .2008

Environmental Defense Fund

6.3 .2008

ETC group

25.3.2008

National Sience Foundation

27.3.2008

Uni Mass Lowell

18.4.2008

20.4.2008

18.6.2008

23.6.2008

23.6.2008

3.9 .2008

Verband der Chemischen Industrie e.V. (VCI)

9.9 .2008 
27 Leitungsmitglied

28 Nanotechnologieverantwortlicher

29 Nanotechnologieverantwortlicher

30 Nanotechnologieverantwortliche

31 Nanotechnologieverantwortliche

32 Nanotechnologiespezialistin

33 Nanotechnologieverantwortlicher

34 Nanotechnologieverantwortlicher

35 Nanotechnologieverantwortliche

36 Nanotechnologiespezialist

37 Leitungsmitglied

38 Nanotechnologieverantwortliche

39 Nanotechnologieverantwortlicher

40 Mitglied der NanoKommission

41 Nanotechnologieverantwortlicher

42 Nanotechnologieverantwortlicher

43 Chemierechtsspezialist

44 Leitungsmitglied

45 Nanotechnologiespezialist

46 Leitungsmitglied

47 Nanotechnologieverantwortliche

48 Nanotechnologieverantwortlicher

49 Nanotechnologieverantwortliche

50 Nanotechnologiespezialist

51 Nanotechnologieverantwortlicher

52 Nanotechnologiespezialist

53 Nanotechnologie/Dialogspezialist

54 Nanotechnologieverantwortlicher

55 Nanotechnologieverantwortliche
Sonderforschungsgruppe Institutionenanalyse (sofia), Hochschule Darmstadt

Evonik Degussa

10.9.2008

Bundesinstitut für Risikobewertung (BfR)

BASF

Bund für Umwelt und Naturschutz, Friends of the Earth Deutschland (BUND)

Rechtsanwältin, Dipl.-Informationswirtin Chemie (FH)

Umweltbundesamt (UBA)

16.9.2008

Bundesanstalt für Arbeitsschutz (BAuA)

18.9.2008

Bundesanstalt für Arbeitsschutz (BAuA)

Öko-Institut e. V.

18.9.2008

19.9.2008

Bundesministerium für Umwelt, Naturschutz und Reaktorsicherheit (BMU)

Bundesministerium für Umwelt, Naturschutz und Reaktorsicherheit (BMU)

Bundesministerium für Umwelt, Naturschutz und Reaktorsicherheit (BMU)

Universität Bremen

29.9.2008

European Environmental Citizens'Organisation for Standardisation (ECOS)

Europäische Kommission, DG Forschung

London School of Economics

7.10 .2008

Bundesinstitut für Risikobewertung (BfR)

10.10 .2008

15.10 .2008

Institut für Ökologische Wirtschaftsforschung (IÖW)

NanoKommission der deutschen Bundesregierung

Bund für Umwelt und Naturschutz, Friends of the Earth Deutschland (BUND)

Europäische Kommission, DG Unternehmen und Industrie

Bundesanstalt für Arbeitsschutz (BAuA)

27.10.2008

Abgeordneter, Europäisches Parlament

30.10 .2008

Europäische Kommission, DG Umwelt

30.10 .2008

nano KTN

31.10 .2008

Demos

4.11.2008

Royal Society

5.11 .2008

Nanotechnology Industries Association

(NIA) 
56 Nanotechnologieverantwortliche

57 Nanotechnologiespezialist

58 Dialogspezialistin

59 Nanotechnologieverantwortliche

60 Nanotechnologieverantwortliche

61 Nanotechnologieverantwortliche

62 Leitungsmitglied

63 Nanotechnologieverantwortliche

64 Nanotechnologiespezialist

65 Mitglied

66 Nanotechnologiespezialist

67 Nanotechnologieverantwortlicher

68 Nanotechnologieverantwortlicher

69 Nanotechnologieverantwortlicher

70 Verantwortlicher

71 Verantwortlicher

72 Nanotechnologieverantwortliche

73 Nanotechnologieverantwortliche

74 Nanotechnologieverantwortliche
Europäische Kommission, DG Unternehmen und Industrie

Department for Environment, Food and Rural Affairs (Defra)

Involve

Europäische Kommission, DG Umwelt

13.11.2008

Europäische Kommission, DG Forschung

17.11.2008

Europäische Kommission, DG Gesundheit

19.11.2008 und Lebensmittelsicherheit

Center for Science and Policy, University of Cambridge

Responsihle Nano Forum

25.11.2008

Greenpeace International, University of Exeter

OECD Working Praty on Nanotechnology, EPFL

University of Sheffield, EPSRC

2.12.2008

Europäische Kommission, DG Unternehmen und Industrie

Nanocap Project

3.12 .2008

Europäische Kommission, DG Gesundheit und Lebensmittelsicherheit

OECD Working Praty on Manufactured Nanomaterials

OECD Working Praty on Nanotechnology

Which

European Chemieals Agency (ECHA)

Health\&Safety Executive (HSE)
19.12.2008

12.1.2009

20.1.2009

4.12.2008

9.12.2008

10.12.2008

26.1.2009 
